\title{
Subcellular Distribution of ${ }^{[25}$ I]Iodoaryl $\beta$-Methyl Fatty Acids
}

\author{
CHARLOTTE A. OTTO,' LAWRENCE E. BROWN² and HELEN LEE ${ }^{2}$
}

${ }^{1}$ University of Michigan-Dearhorn. Dearborn. MI 48128 and 'University of Michigan Medical Center. Ann Arbor. MI 48109. L.S.A.

\author{
(Receired 15 Mardh 1985)
}

\begin{abstract}
Subcellular distribution studies of two aryl branched-chain and one aryl straight-chain iodinated fatty acids were carried out as part of a continuing effort to determine if such acids are metabolized by $\beta$-oxidation. For the (1)-iodoaryl fatty acids, a change in subcellular radioactivity location was observed which was chain-length dependent. Chain lengths of 15 carbons. straight and branched, were largely found in the nuclear-membrane fraction, whereas a chain length of 8 carbons was largely located in the cytosol. No unequivocal evidence for metabolic trapping in the mitochondria was observed for $\omega$-iodoaryl branched-chain fatty acids using the centrifugation technique employed in this study.
\end{abstract}

\section{Introduction}

Several research groups have investigated the use of radiolabelled branched-chain fatty acids as potential myocardial imaging agents. ${ }^{11-4)}$ Studies with $\beta$-methyl and $\beta . \beta$-dimethyl iodoalkyl fatty acids served to confirm that rapid deiodination occurred independent of the alkyl structure."(1) Studies using ${ }^{\text {" } C-~} \beta$-methylheptadecanoic acid in rats and dogs showed a prolonged myocardial retention of radioactivity.2.4) Stabilization of the radioiodide label by incorporation of iodine onto an aryl ring has also been examined. It was found that $\omega$-iodoaryl. $\beta$-methyl branched-chain fatty acids were also extracted efficiently by the myocardium and possessed prolonged retention in the heart. ${ }^{(1.3)}$

Monoalkylation at the $\beta$ position of a fatty acid should yield a fatty acid (1) capable of transport. barring steric hindrance, into the mitochondria where $\beta$-oxidation occurs. (2) capable of dehydrogenation followed by hydration. the first two steps of $\beta$-oxidation. to yicld a $\beta$-hydroxy, $\beta$-methyl acid. and (3) incapable of a second dehydrogenation to yield a $\beta$-keto acid. It has been suggested that the $\beta$-hydroxy, $\beta$-methyl "C-fatty acid. the theoretical product of dehydrogenation then hydration, is trapped in the mitochondria and that this metabolic trapping is responsible for prolonged retention of radioactivity in the myocardium.

This prolonged retention for both the "C- $\beta$-methyl fatty acid and the radioiodinated (o-iodoaryl, $\beta$-methyl fatty acids suggests a similarity in biochemical behavior. If metabolic trapping occurs for radioiodinated branched-chain acids, one could postulate an increase in radioactivity in the mitochondria with time. In order to evaluate this possibility and to obtain further information useful in interpreting im- ages obtained with $\omega$-iodoaryl- $\beta$-methyl branched fatty acids, the distribution of radioactivity in various cellular fractions at two time intervals was obtained for two branched-chain acids and, for purposes of comparison. one straight-chain $\omega$-iodoaryl acid.

\section{Materials and Methods}

Sprague-Dawley female rats weighing $250-350 \mathrm{~g}$ were used. They were given rat chow and water ad libitum, and kept under conditions of alternating 12-h periods of light and dark. Rats were sacrificed by decapitation after ether anesthesia.

\section{Radiolabelled compounds}

The radioiodinated fatty acids included in this study were (1) 15-( $p-\left[{ }^{125} \mathrm{I}\right] \mathrm{I}$ iodophenyl)-3-methylpentadecanoic acid (15-IP $\beta$ M). (2) 8-( $p$-[25)I]iodophenyl)3-methyloctanoic acid (8-IP $\beta M$ ), and (3) $15-\left(p-\left[{ }^{125} I\right]\right.$ iodophenyl)pentadecanoic acid (IPPA). They were prepared as previously described. (i)

\section{Analysis of the heart}

Heart analysis was performed at 5- and 40-min post injection for IPPA, 15-IP $\beta$ M, and 8-IP $\beta$ M. Four rats were killed per each time interval per each fatty acid. $\Lambda$ fter sacrifice the hearts were promptly excised, rinsed free of blood in ice-water and cut into thirds. The thirds were maintained between 0 and 4 C. removed and counted for $I$ min and immediately returned to a $0-4 \mathrm{C}$ ice-water bath. The 1 -min counts were used as $100^{\circ}{ }_{\circ}$ values for determining total recovery. All succeeding procedures were performed at $0-4 \mathrm{C}$. The heart thirds were recombined and homogenized using an isotonic sucrose-phosphate buffer ( $\mathrm{pH} 7.0$ at $0 \mathrm{C}$ ). The homogenates were centrifuged at $650 \mathrm{~g}$ for $5 \mathrm{~min}$, the supernatant $S_{0}$ was 


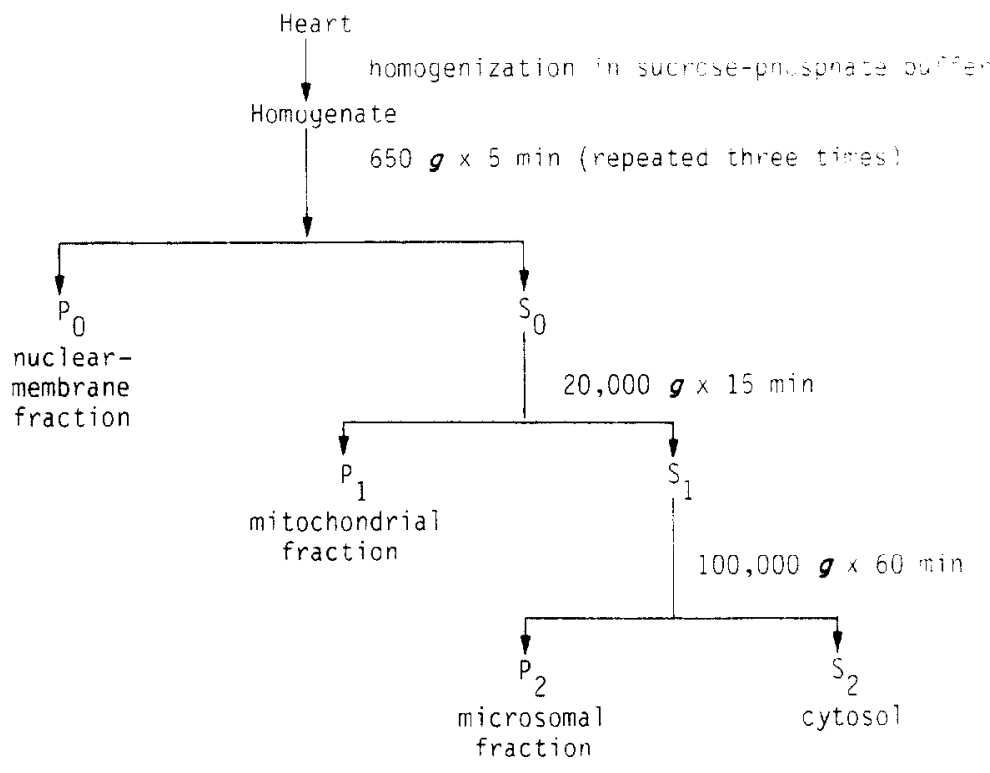

Fig. 1. Centrifugation procedure for isolation of subcellular fractions.

removed and held between 0 and $4^{\circ} \mathrm{C}$. The pellet, $P_{0}$, was resuspended using sucrose-phosphate buffer as above and recentrifuged at $650 \mathrm{~g}$ for $5 \mathrm{~min}$. This washing process was repeated two times. Supernatant fractions $\left(S_{0}\right)$ from the $650 \mathrm{~g}$ centrifugations were combined and centrifuged at $20,000 \mathrm{~g}$ for $15 \mathrm{~min}$ to yield pellet $P_{1}$ and supernatant $S_{1}$. Supernatant $S_{1}$ was removed and centrifuged at $100,000 \mathrm{~g}$ for $60 \mathrm{~min}$ yielding supernatant $S_{2}$ which was decanted, and final pellet, $P_{2}$. Figure 1 is a diagrammatic summary of the centrifugation procedure. Each pellet, $P_{0}, P_{1}$, and $P_{2}$ was treated as a single sample for counting using an autogamma scintillation counter. $S_{2}$ was divided into approximate thirds for counting. All values were corrected for radioactive decay, counting efficiency, and background.

\section{Results and Discussion}

The centrifugation procedure employed for separation of various subcellular fractions was slightly modified from that published by Lighty and Bertrand $^{(5)}$ for beef heart. Similar centrifugation pro- cedures have been used for isolating mitochondria and other subcellular fractions from rat heart. ${ }^{(6)}$ The distribution of radioactivity in the nuclearmembrane, mitochondrial, microsomal and cytosolic fractions at 5 and $40 \mathrm{~min}$ for IPPA, 15-IP $\beta$ M and 8-IP $\beta M$ is presented in Table 1. Total percent recovery ranged from 82 to $90 \%$. There are significant differences in subcellular radioactivity distribution among these compounds.

The percent of activity in the mitochondria is higher for IPPA and for its $\beta$-methyl analog, 15-IP $\beta \mathrm{M}$, than for 8-IP $\beta \mathrm{M}$. Coenen and colleagues have shown that $15-\left(p-\left[{ }^{79} \mathrm{Br}\right]\right.$ bromophenyl)pentadecanoic acid, BPPA, is metabolized. (7) Products of $\beta$-oxidation, $\quad p$-bromobenzoic acid and 3 -( $p$-bromophenyl)propanoic acid, were isolated from heart tissue. IPPA is also expected to metabolize by $\beta$-oxidation and some concentration of radioactivity in the mitochondria is thus expected. The retention of radioactivity in the nuclear-membrane fraction for IPPA is expected, based on data from BPPA which indicates that loss of activity from phospholipids or polar substrates is slow from 0.5 to

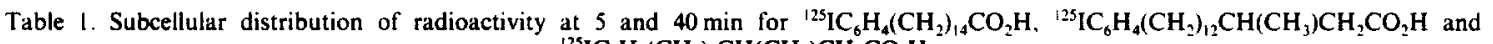

\begin{tabular}{|c|c|c|c|c|c|c|}
\hline \multirow[b]{2}{*}{ Compound } & \multirow[b]{2}{*}{$\begin{array}{l}\text { Time } \\
(\min )\end{array}$} & \multicolumn{5}{|c|}{ Fraction $(\%)^{a}$} \\
\hline & & $\begin{array}{l}\text { Nuclear- } \\
\text { membrane }\end{array}$ & Mitochondrial & Microsomal & Cytosolic & Recovery \\
\hline${ }^{125} \mathrm{IC}_{6} \mathrm{H}_{4}\left(\mathrm{CH}_{2}\right)_{14} \mathrm{CO}_{2} \mathrm{H}$ & 5 & $58.5 \pm 3.7$ & $13.4 \pm 1.7$ & $8.3 \pm 1.1$ & $19,9 \pm 1.3$ & $90.1 \pm 1.0$ \\
\hline (IPPA) & 40 & $60.1 \pm 1.7$ & $8.9 \pm 1.6$ & $7.7 \pm 0.8$ & $20.8 \pm 1.1$ & $88.0 \pm 3.0$ \\
\hline${ }^{25} \mathrm{IC}_{6} \mathrm{H}_{4}\left(\mathrm{CH}_{2}\right)_{12} \mathrm{CH}\left(\mathrm{CH}_{3}\right) \mathrm{CH}_{2} \mathrm{CO}_{2} \mathrm{H}$ & 5 & $72.1 \pm 0.5$ & $13.7 \pm 0.3$ & $3.7 \pm 0.2$ & $10.4 \pm 0.2$ & $86.7 \pm 1.2$ \\
\hline$(15-1 \mathrm{P} \beta \mathrm{M})$ & 40 & $67.9 \pm 0.6$ & $13.6 \pm 0.9$ & $3.5 \pm 0.4$ & $14.8 \pm 1.2$ & $81.6 \pm 4.2$ \\
\hline $122 \mathrm{IC}_{6} \mathrm{H}_{4}\left(\mathrm{CH}_{3}\right)_{6} \mathrm{CH}\left(\mathrm{CH}_{3}\right) \mathrm{CH}_{2} \mathrm{CO}_{2} \mathrm{H}$ & 5 & $35.4 \pm 1.4$ & $7.6 \pm 0.5$ & $3.7 \pm 0.3$ & $55.3 \pm 2.5$ & $88.0 \pm 2.5$ \\
\hline$(8-\mathrm{IP} \beta \mathrm{M})$ & 40 & $39.4 \pm 1.6$ & $7.5 \pm 0.7$ & $3.2 \pm 0.1$ & $50.1 \pm 1.5$ & $82.2 \pm 1.7$ \\
\hline
\end{tabular}

"Data based on four hearts per compound per time interval. All values reported as mean \pm SE. 
$10 \mathrm{~min}$. The IPPA nuclear-membrane fraction should contain most of the phospholipid/polar substrate activity observed for BPPA.

The similarity in mitochondrial activity levels for IPPA and its $\dot{\beta}$-methyl analog at $t=5 \mathrm{~min}$ suggests that carnitine-mediated transport into the mitochondria is not inhibited by the $\beta$-methyl substituent. The reduction by half of mitochondrial activity for the 8 carbon $\beta$-methyl fatty acid, 8 -IP $\beta \mathrm{M}$, may reflect passive diffusion between cytosol and mitochondria for shorter chain fatty acids rather than hindered transport.

The activity level in the mitochondria may be at a minimum. The technique used in this study, immediate immersion of dissected heart into saline held at $0-4 \mathrm{C}$ and performance of all subsequent steps at $0-4 \mathrm{C}$. would not completely prevent metabolism. The total length of time between dissection and separation of mitochondria $P_{1}$ from supernatant $S_{1}$ did not exceed $60 \mathrm{~min}$. Storage of dog heart tissue at room temperature for $60 \mathrm{~min}$ reduced the amount of cholesteryl esters but had no apparent effect on triglycerides and phospholipids. ${ }^{(8)}$ Because of the short time span and the reduced metabolic rates at $0-4 \mathrm{C}$ the changes in radioactivity location due to metabolism are expected to be minimal. An accurate assessment of cellular distribution would require microautoradiography coupled with electron microscopy as described. ${ }^{191}$

The location of the majority of radioactivity varies with chain length. For the 15 carbon IPPA, most of the activity at both 5 and $40 \mathrm{~min}$ is associated with the $650 \mathrm{~g}$ nuclear-membrane fraction. This is also true for the 15 carbon $\beta$-methyl analog of IPPA, except that a higher percentage $\left(\sim 70 \mathrm{vs} \sim 60^{\circ}\right.$ ) is associated with the $650 \mathrm{~g}$ fraction. This distribution of radioactivity is in sharp contrast to that of $8-\operatorname{IP} \beta M$ for which $50-55^{\circ}{ }_{0}$ of activity at 5 and $40 \mathrm{~min}$ is associated with the cytosolic fraction and only $35-40 \%$ of activity is associated with the $650 \mathrm{~g}$ nuclear-membrane fraction. The similarity of radioactivity distribution between IPPA and its $\beta$-methyl analog suggests that the differences observed for the 8 carbon $\beta$-methyl compound are due to the shorter chain length.

The distribution of radioactivity does not support trapping of the aryl $\beta$-methyl fatty acids in the mitochondria. The percent of activity associated with the mitochondrial fraction was experimentally equivalent at both 5 and $40 \mathrm{~min}$ for both $15-\mathrm{IP} \beta \mathrm{M}$ and $8-I P \beta M$. If it is assumed (1) that most fatty acids extracted by the myocardium are eventually metabolized by the mitochondria. (2) that transport of the $\beta$-hydroxy. $\beta$-methyl fatty acid, the presumed metabolite. out of the mitochondria does not occur and (3) that the transport process itself is unaffected by a metabolic bottleneck within the mitochondria. then increasing amounts of mitochondrial radioactivity with increasing time post-injection would be expected. This was clearly not demonstrated. The data presented here do not preclude an increase, then a decrease, in radioactivity between 5 and $40 \mathrm{~min}$ with a fortuitous coincidence of values for both $15-\mathrm{IP} / \mathrm{M}$ and 8 -IP $\beta$ M. Further work at more time intervals and with acids of high specific activity are necessary. Because of possible differences in lipophilicity between aryl and alkyl fatty acids, the alkyl $\beta$-methyl fatty acids may possess a different subcellular distribution and may show evidence for metabolic trapping. The alkyl $\beta$-methyl acids do show a different time course of radioactivity in the heart. An increase in radioactivity values at 15 and $60 \mathrm{~min}$ relative to 5 min has been reported in rat. ${ }^{(4)}$ The aryl $\beta$-methyl acids have constant levels of radioactivity over this same time frame. Different behavior may therefore be expected.

It must be noted that the percent values in Table 1 for $15-\mathrm{IP} \beta \mathrm{M}$ and $8-\mathrm{IP} \beta \mathrm{M}$ at 5 and $40 \mathrm{~min}$ can be directly compared as both acids have essentially constant percent dose per organ values in the myocardium from 5 to $40 \mathrm{~min} .^{11.10)}$ The third acid, IPPA, has an approximate $55 \%$ decrease in percent dose per organ values from 5 to $40 \mathrm{~min}^{(1)}$ and this decrease is not directly incorporated into the data of Table 1. There should be no effects on the percent distribution due to differences in specific activity as all fatty acids used had a specific activity of about $1 \mathrm{mCi} / \mathrm{mmol}$. Smaller doses of very high specific activity fatty acids may show differing (lower) mitochondrial relative concentrations. This is particularly true if steadystate conditions in terms of transport in and out of the mitochondria were achieved in the work reported here.

The finding of the majority of radioactivity for IPPA and $15-1 \mathrm{P} \beta \mathrm{M}$ in the $650 \mathrm{~g}$, nuclear-membrane fraction suggests that the fatty acids have been incorporated into phospholipids. This is in agreement with results published for BPPA. Of $57 \%$ of the extracted organic activity, $17.1 \%$ consisted of phospholipids or polar substrates and only $2.3_{\mathrm{o}}^{\circ}$ consisted of triglycerides. ${ }^{(7)}$ These findings are directly contradicted by another study using IPPA which showed that $65 \%$ of the IPPA was found in the cardiac triglycerides. (1) As an analysis of the radioactive compounds was not done in this study, direct comparisons with these other studies cannot be done. It should be further noted that the protocols for all of these studies were different which may explain the apparently contradictory results.

In conclusion, there is evidence for chain-length dependence in the distribution of radioactivity within the myocardial cell for the $\omega$-iodoaryl $\beta$-methyl fatty acids. This is in addition to the previously demonstrated myocardial radioactivity level dependence on chain length." As the alkyl portion of these acids is reduced from 15 to 8 carbons, the majority of radioactivity shifts in location from the nuclear-membrane fraction to the cytosol. The data presented here together with other reported data ${ }^{(1.7 .10)}$ suggest that the aryl $\beta$-methyl fatty acids do not appear to be 
substrates for $\beta$-oxidation, nor do they appear to accumulate in the mitochondria by the $\beta$-oxidation process.

Acknowledgements - Research was supported by Grant No. HL 25459-03 from the National Heart, Lung and Blood Institute, by D.O.E. Contract No. DE-ACO-2-76EV02031.

The authors are particularly grateful to $F$. F. Knapp, Jr and M. M. Goodman at Oak Ridge National Laboratory for the gift of $15-I P \beta M$.

The authors thank K. Lapanowski-Netzel for technical assistance and I. Sayer for help in preparing the manuscript.

\section{References}

1. Otto C. A., Brown L. E. and Scott A. M. J. Nucl. Med. 25, 75 (1984).

2. Livni E., Elmaleh D. R., Levy S., Brownell G. L. and Strauss H. W. J. Nucl. Med. 23, 169 (1982).
3. Goodman M. M.. Kirsch G. and Knapp F. F. J.$J$ Med. Chem. 27, 390 (1984).

4. Elmaleh D. R., Livni E., Levy S.. Varnum D., Strauss H. W. and Brownell G. L. Int. J. Nucl. Med. Biol. 10, 181 (1983).

5. Lighty G. W. Jr and Bertrand H. A. Anal. Biochem. 99. 41 (1979).

6. Christophersen B. O. and Bremer J. Biochim. Biophis. Acta 280, 506 (1972).

7. Coenen H. H.. Harmand M.-F., Kloster G. and Stocklin G. L. J. Nucl. Med. 22, 891 (1981).

8. Van Der Vusse G. J., Roemen T. H. M. and Reneman R. S. Biochim. Biophys. Acta 617, 347 (1980).

9. Harris P., Gloster J. A. and Ward B. J. Arch. Mal Coeur 73, 593 (1980).

10. Goodman M. M., Kirsch G. and Knapp F. F. Jr. J. Labelled Compd. Radiopharm. 19, 1316 (1982).

11. Chien K. R., Han A., White J. and Kulkarni P. Am. J. Physiol. H693 (1983). 